\title{
On the interdependence of temporal and spatial judgments
}

\author{
YIH LERH HUANG \\ Memorial University, St. John's, Newfoundland, Canada \\ and \\ BILL JONES \\ Carleton University, Ottawa, Ontario, Canada
}

\begin{abstract}
Three experiments are reported on the tau and kappa effects, the dependence of judgments of distance upon duration (tau) and of judgments of duration upon distance (kappa). In Experiment 1 , three lights in a horizontal sequence were used to define two temporal and two spatial intervals over a total duration of $160 \mathrm{msec}$. The subject was required to choose the shorter of either the two durations or the two distances. The results confirmed Collyer's (1977) findings that the two effects are inconsistently observed across subjects when the display duration is brief. In Experiment 2, display duration was systematically manipulated from 160 to $1,500 \mathrm{msec}$. It is argued that relative temporal judgments should become easier as the total display duration is increased and that, hence, the kappa effect should become less marked. On the other hand, relative spatial judgments should become more difficult as the total duration of the display is increased, and the tau effect should become more marked. The data were in conformity with the hypothesis. In Experiment 3, data are presented for a tau experiment which fit the assumption that the effect depends upon a weighted average of distance and the expected distance which would be traversed in the given time at constant velocity.
\end{abstract}

In his classic demonstrations, Helson (1930; Helson \& King, 1931) stimulated three points in succession on a subject's forearm to define two spatial extents $\left(s_{1}\right.$ and $\left.s_{2}\right)$ and two temporal durations $\left(t_{1}\right.$ and $\left.t_{2}\right)$. He found that relative judgments of the two extents were highly correlated with the relative durations; that is, subjects tended to judge that $s_{1}$ was the longer of the two extents when $t_{1}$ was the greater duration and to judge that $s_{1}$ was the shorter when $t_{2}$ was the smaller duration. Helson called this phenomenon the tau effect (see also Bill \& Teft, 1969, 1972; Collyer, 1977). In the same way, a longer (or shorter) temporal interval can be made to appear shorter (or longer) by decreasing (or increasing) the associated spatial interval. Following Cohen, Hansel, and Sylvester (1953, 1954, 1955), we call this second effect the kappa effect (see also Price-Williams, 1954). The literature on the two effects has been reviewed by Jones and Huang (1982).

The purpose of the present studies was to establish

This research formed a part of the first author's doctoral thesis at Carleton University carried out under the supervision of the second author. We would like to thank the examining board, L. Allan, S. Jones, J. Kelly, W. M. Petrusic, and W. Webster for their stimulating comments. Requests for reprints should be directed to Bill Jones, Department of Psychology, Carleton University, Ottawa, Ontario K1S 5B6, Canada. The first author is now affiliated with the Faculty of Engineering and Applied Science at Memorial University. the boundary conditions for the tau and kappa effects (Experiments 1 and 2) and to provide evidence for a descriptive model of the tau effect (Experiment 3) which has been shown to apply reasonably well to the kappa effect (Jones \& Huang, 1982).

Collyer (1977) has provided some information on the lower temporal and spatial bounds of the tau and kappa effects. In fact, Collyer's seems to be the only published failure to observe consistent tau and kappa effects using the three-stimulus paradigm. Collyer fixed the total temporal duration (T) of a visual display at $160 \mathrm{msec}$ and the spatial extent (S) at $4 \mathrm{deg}$ of visual angle and presented temporal and spatial intervals by means of three successive 10 -msec flashes. Methodologically there were a number of variables which, as Collyer noted, may have accounted for his failure to observe tau and kappa. Perhaps most importantly, the value of $T$ was very brief compared with other published studies which have used values in the second rather the millisecond domain (e.g., Bill \& Teft, 1969, 1972; Cohen et al., 1953, 1954, 1955; Helson \& King, 1931; Price-Williams, 1954).

Since the occurrence of tau and kappa may depend upon the lower limits of the display duration, one purpose of the present study was to investigate systematically the effect of $T$ on both effects. Experiment 1 was essentially a slightly modified replication of Collyer's (1977) study. With brief values of T, eye movements, or eye-movement tendencies, perhaps 
conditioned by reading habits, may influence perceptual accuracy (e.g., Crovitz \& Daves, 1962). Bill and Teft (1969), using an adjustment method, found no effect of display direction on the tau effect. However, they used displays varying in duration from 1 to $7 \mathrm{sec}$. Since eye-movement tendencies may be more crucial when $T$ is brief, we varied display direction, left to right or right to left. In Experiment 2, $T$ was varied from 160 to $1,500 \mathrm{msec}$. For both experiments, the mean proportion of "second-intervalshorter" responses was plotted against either the temporal or the spatial variable according to the required judgment. Positive slopes of the plots would indicate the presence of the tau or kappa effects.

\section{EXPERIMENT 1}

\section{Method}

Subjects. Three subjects (one male and two females) with normal or corrected-to-normal vision served in both spatial and temporal conditions and were paid \$2/h.

Apparatus. A Data General Nova 1220 computer controlled the timing and generation of signals, which were presented by light-emitting diodes (Texas Instruments TIL 209) mounted behind a clear plastic board. A video monitor positioned behind the signal display relayed all other instructions. The viewing distance was about $1 \mathrm{~m}$, and the display subtended $4 \mathrm{deg}$ of visual angle.

Design and Procedure. On each trial, the subject observed a sequence of three lights, the first and the third of which were fixed at 4 deg of visual angle, that is, $S=s_{1}+s_{2}=4$ deg of visual angle. The five values of $s_{1}$ were $.67,1.33,2.00,2.67$, and $3.33 \mathrm{deg}$. The total duration was fixed at $160 \mathrm{msec}\left(T=t_{1}+t_{2}=160 \mathrm{msec}\right)$. The five values of $t_{1}$ were $20,56,80,104$, and $140 \mathrm{msec}$. The duration of each signal was $10 \mathrm{msec}$. On each trial, $s_{1}$ and $t_{1}$ were sampled without replacement at random. During each block of 250 trials, there were 10 replications for each of the $25 s_{1}-t_{1}$ combinations. Fifteen practice trials were presented at the beginning of each block and were excluded from analysis. Each subject took part in 20 blocks of trials, with 5 blocks for each display direction (left to right and right to left), under spatial and temporal conditions. The subjects thus participated in a total of 5,000 trials.

Each trial proceeded as follows. The word "READY" appeared on the video monitor, and the subject pressed any button to initiate the stimulus display. After a $500-\mathrm{msec}$ delay, the stimulus sequence was presented and the subject responded by pressing one of two response buttons to indicate whether the first or second spatial (or temporal) interval appeared the shorter. A third button was available to the subject to indicate that the display was unclear. This response was rarely made, and such trials were excluded from analysis.

Daily sessions consisted of two to four blocks of trials with rest periods. Temporal and spatial conditions were run on alternate days.

\section{Results}

Figures 1 and 2 plot the mean proportion of responses in which the subject indicated that the second (spatial or temporal) interval was the shorter for each $s_{1}-t_{1}$ combination in the spatial judgment condition (tau) and the temporal judgment condition (kappa), respectively. The two display directions were combined in the plots since ANOVA showed no significant effect of display direction (cf. Bill \& Teft, 1969).

For the tau experiment, two statistically significant

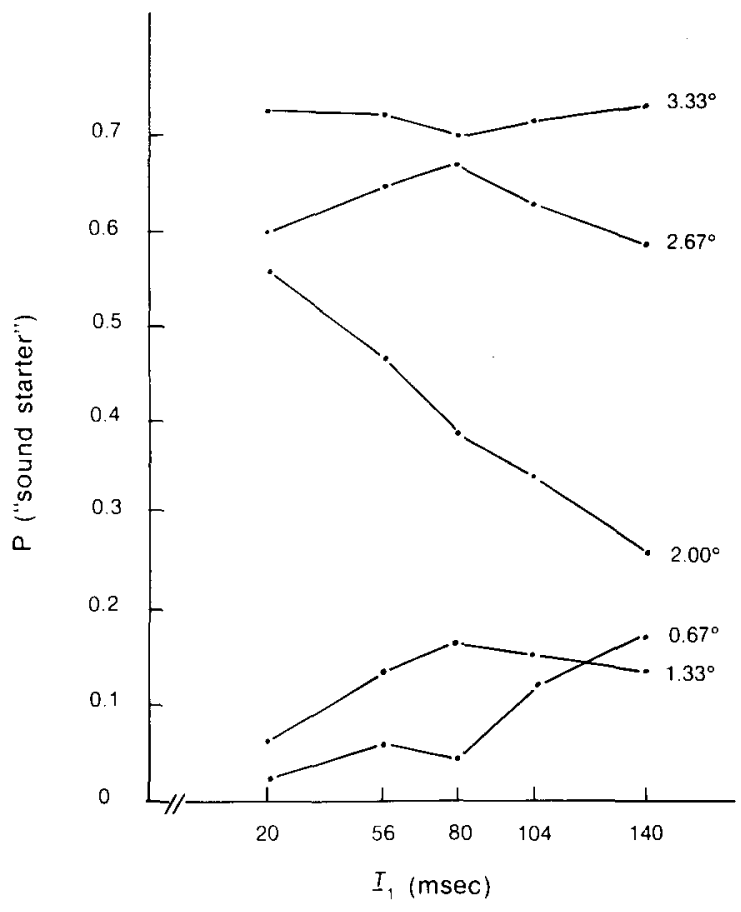

Figure 1. Mean proportion (P) of "second-shorter" responses for the tau experiment as a function of $t_{1}$ (msec) for each level of $s_{1}(.67$ to 3.33 deg).

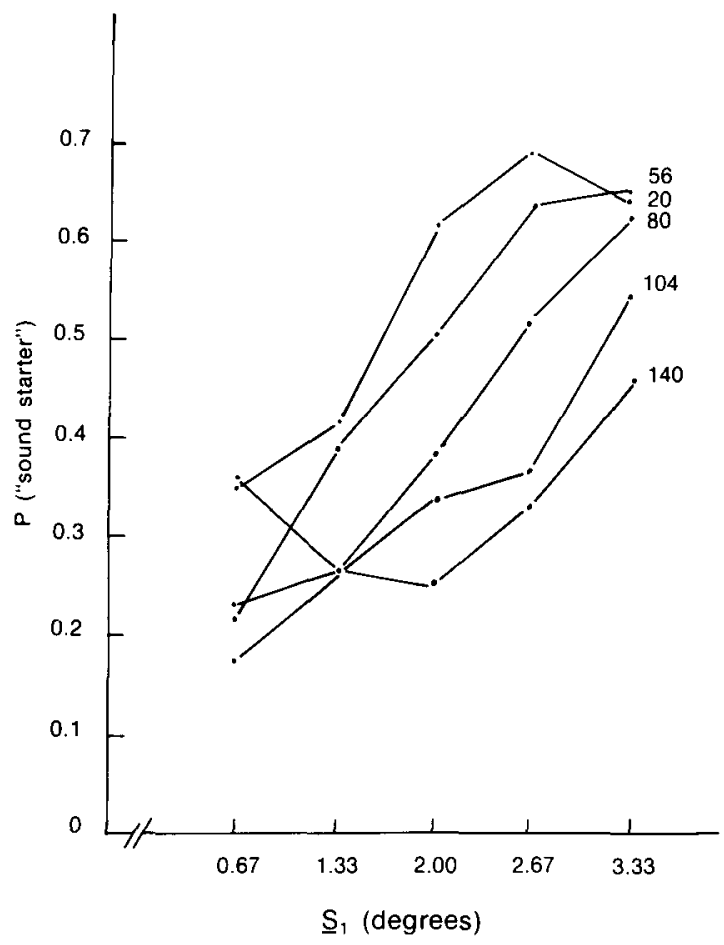

Figure 2. Mean proportion (P) of "second-shorter" responses for the kappa experiment as a function of $s_{1}$ (degrees) for each level of $t_{1}$ (20 to $140 \mathrm{msec}$ ). 


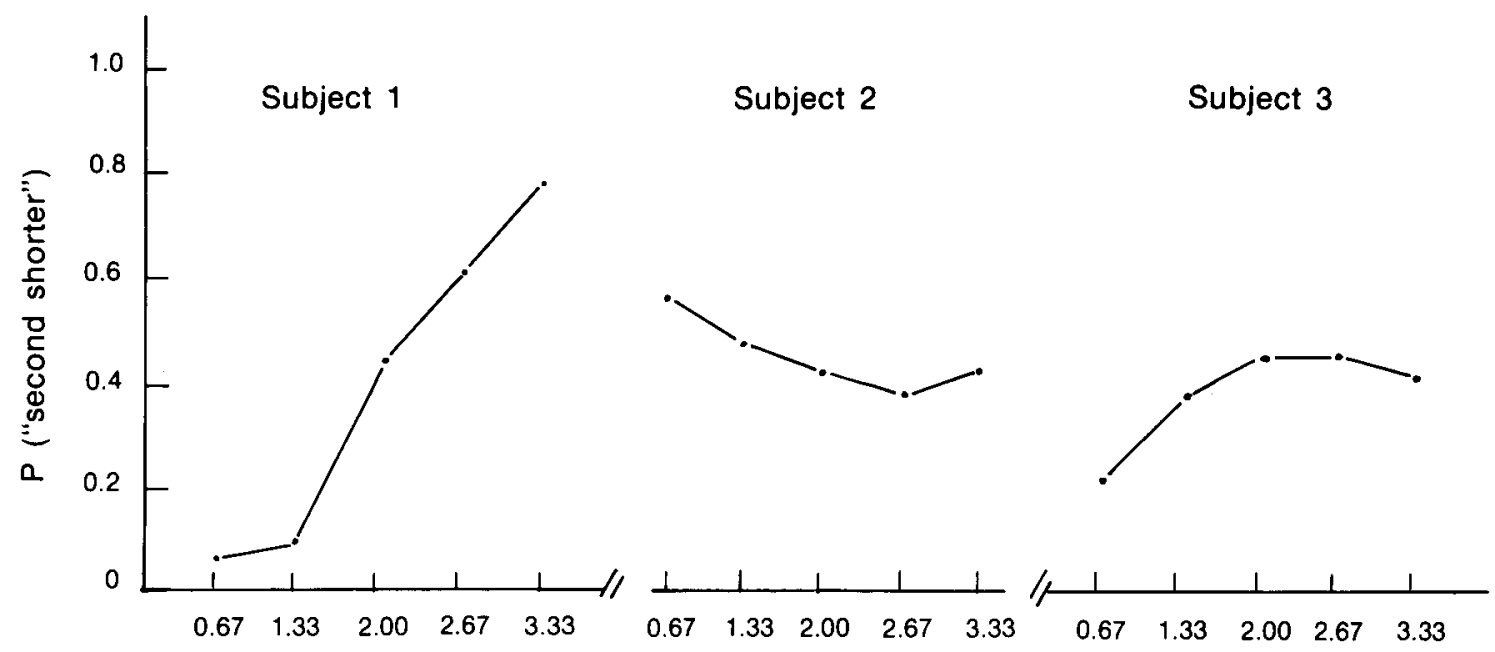

Figure 3. Mean proportion (P) of "second-shorter" responses for the kappa experiment for individual subjects collapsed across the levels of $t_{1}$.

effects were observed: $s[F(4,8)=22.96, p<.001]$ and the $s \times t$ interaction $[F(16,32)=6.92, p<.001]$. Figure 1 shows good separation between the five curves, indicating that the subjects discriminated, reasonably well between the five distances, although there is some indication of a negative time-order error (the overestimation of the second of a pair of successive stimuli).

Figure 1 also shows that the slopes of the curves change as $s_{1}$ increases. Positive slopes would, of course, indicate the tau effect. Only the lower two curves $\left(s_{1}<s_{2}\right)$ show any increasing trend. When $s_{1}>s_{2}$ (the upper two curves), there is no indication of temporal influence on the spatial judgment. When $s_{1}=s_{2}$, the curve is clearly decreasing, indicating the reverse of the tau effect.

For the kappa experiment (Figure 2), ANOVA showed no significant main effects, indicating the absence of any systematic kappa effect. However, Figure 3 plots mean proportions as a function of $s_{1}$ collapsed over the $t_{1}$ values and shows the degrees of variability between subjects. Subject 1 shows a kappa effect, Subject 2 a reverse kappa, and Subject 3 a tendency for the curve to flatten as $s_{1}$ increases. The averages presented in Figure 2 may, therefore, be misleading. Each of the three subjects shows a quite different effect.

In other words, we found no evidence for consistent tau or kappa effects when the total duration of the display was relatively brief (cf. Collyer, 1977).

\section{EXPERIMENT 2}

No previous experiments seem to have investigated the effects of systematic variation in $T$ in the lower range of values on the occurrence of tau and kappa, although Cohen et al. (1954) did examine the range .6 to $6.4 \mathrm{sec}$ for the kappa effect. In the present experiment, $T\left(T=t_{1}+t_{2}\right)$ was varied from 160 to $1,500 \mathrm{msec}$, with fixed spatial extent $\left(\mathrm{S}=\mathrm{s}_{1}+\mathrm{s}_{2}\right)$.

The theoretical significance of varying $T$ is that systematic changes in the discriminability of the two temporal and spatial intervals should result. With fixed $S$, discrimination of extent may be easier when $T$ is relatively brief, since, in order to code distance, the subject must remember the location of the first light and it is plausible to expect that location memory becomes more diffuse over time (Kinchla \& Smyzer, 1967). On the other hand, relative temporal judgments may become more difficult as $\mathrm{T}$ decreases (see Allan, 1979). If the tau and kappa are illusions which arise when the spatial or temporal judgment is indeterminate, varying $T$ is equivalent to varying the "context" of the illusion. We hypothesized, therefore, that the tau and kappa effects should be more marked when the primary discrimination is more difficult and the subject must rely on secondary cues. If spatial judgments become more difficult as $T$ increases, a greater tau effect should be observed with $T$ long; and if temporal judgments are more difficult with $\mathrm{T}$ short, the kappa effect should be more marked in this range.

\section{Method}

Subjects. One male and two females served as subjects in the spatial judgment condition. Two males and one female served as subjects in the temporal judgment condition. All subjects were naive as to the experimental hypotheses. All were paid \$2/h.

Apparatus. The apparatus was that used in Experiment 1.

Design and Procedure. The basic design and procedure were as in Experiment 1. All displays were from left to right. There were eight total durations (T): $160,320,480,640,800,1,000,1,200$, and $1,500 \mathrm{msec}$. For each duration, a set of five values of $t_{1}$ was selected such that $t_{1} / T=.3, .5, .5, .6$, or .7 . The ratio $t_{1} / T$ will 
be referred to as the time factor. For all $\mathrm{T}$ conditions, $\mathrm{S}$ was fixed at $8 \mathrm{deg}$ of visual angle and a set of five $s_{1}$ values was selected such that $s_{1} / S=.3, .4, .5, .6$, or .7 (space factor). Each block of trials, with $T$ fixed, consisted of 10 replications of the $25 s_{1}-t_{1}$ combinations. There were 15 practice trials at the beginning of each block which were excluded from analysis. Five such blocks of trials were randomized in groups of eight, with each group made up of a block from each of the $T$ conditions. Three subjects made spatial judgments and three subjects made temporal judgments on successive weekdays for two to four blocks per day. Each subject participated in a total of 10,000 trials.

\section{Results}

Figure 4 plots the mean proportions of "second shorter" responses collapsed across the values of $T$ for the tau and kappa experiments.

For the tau experiment, the ANOVA showed significant main effects of space $[F(4,8)=3.56, p<.001]$ and time $[F(4,8)=4.03, p<.05]$ and a significant space $\times$ time interaction $[F(28,56)=5.69, p<.001]$. Figure 4 demonstrates the existence of a tau effect for $s_{1} \geqslant s_{2}$ (i.e., $s_{1} / S=.5, .6$, and .7). For $s_{1}<s_{2}$ (i.e., $s_{1} / S=.3$ and .4), there is no evidence of a systematic tau effect.

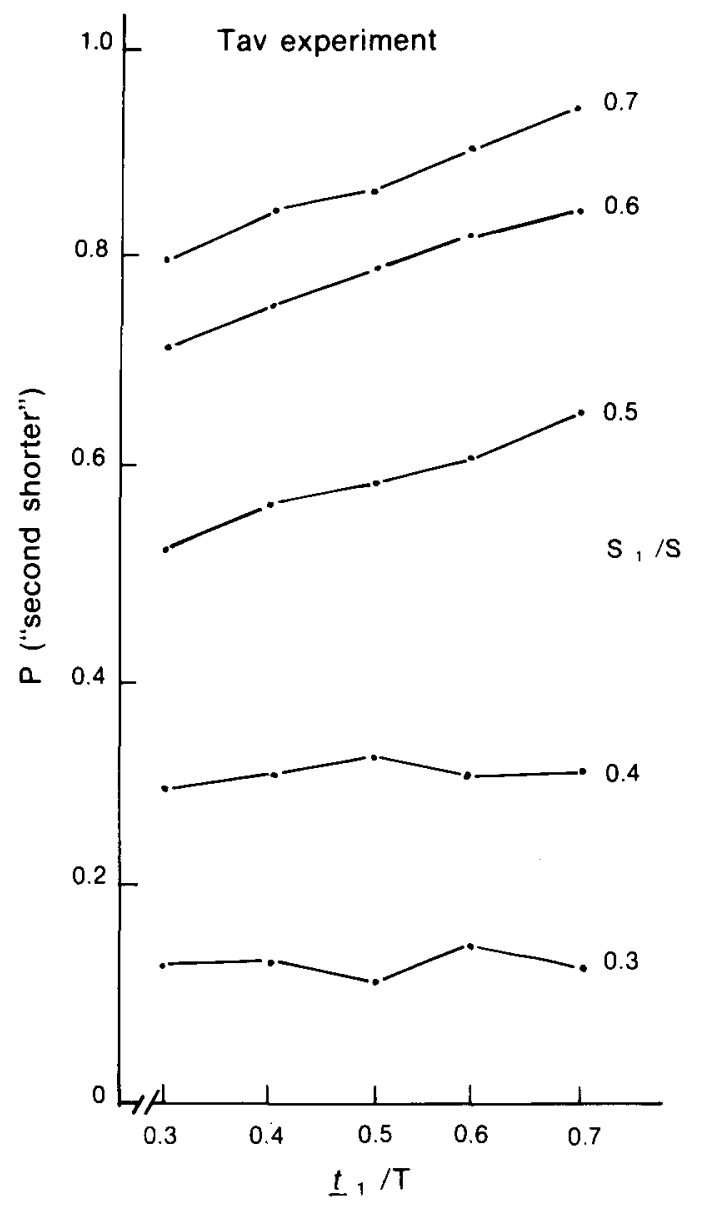

The $\mathrm{T} \times$ Space interaction was also significant for the tau experiment $[\mathrm{F}(28,56)=3.51, \mathrm{p}<.001]$. Table 1 gives the mean proportions for each $s_{1}$ at each value of $T$ collapsed across $t_{1}$ values; that is, Table 1 reflects the change in the influence of $t_{1}$ as $T$ changes. In general, $T$ increases from 160 to $1,500 \mathrm{msec}$, and mean proportions increase for $s_{1}<s_{2}$ but decrease for $s_{1}>s_{2}$.

For the kappa experiment, the main effects of time $[F(4,8)=58.78, p<.001]$ and space $[F(4,8)=16.53$, $\mathrm{p}<.001]$ were significant. The following interactions were also significant: space $\times$ time $[F(112,224)$ $=1.52, \mathrm{p}<.001], \mathrm{T} \times$ space $[\mathrm{F}(28,56)=13.73, \mathrm{p}<$ $.001], \mathrm{T} \times$ time $[\mathrm{F}(28,56)=16.21, \mathrm{p}<.001]$, and $\mathrm{T} \times$ space $\times$ time $[\mathrm{F}(112,224)=1.52, \mathrm{p}<.005]$.

Figure 4 shows clearly both the time and space main effects, the latter indicating the kappa effect. The curves appear roughly parallel, and the space $x$ time interaction, which is relatively trivial in relation to the number of degrees of freedom, does not seem systematic. The importance of parallelism in the plots will be discussed below in connection with Experiment 3.

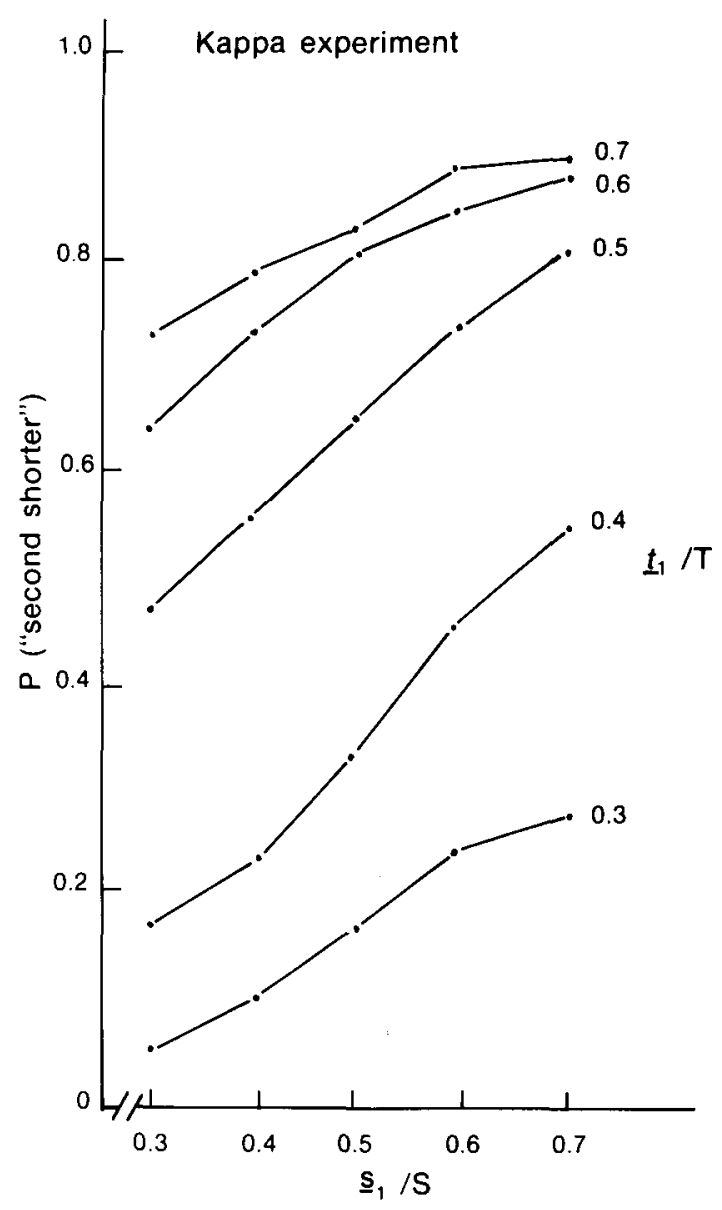

Figure 4. Mean proportion (P) of "second-shorter" responses for temporal and spatial judgments as a function of $s_{1} / S$ and $t_{1} / T$. 
Table 1

Mean Proportions of "Second Shorter" Responses for the Tau Experiment at Each Value of $s_{1} / S$ and $T$ Summarized Across $t_{1} / T$ Conditions

\begin{tabular}{|c|c|c|c|c|c|c|c|c|c|c|c|c|}
\hline \multirow{3}{*}{$\begin{array}{c}\text { T (in } \\
\text { Milliseconds) }\end{array}$} & \multicolumn{10}{|c|}{$s_{1} / S$} & & \\
\hline & \multicolumn{2}{|c|}{.3} & \multicolumn{2}{|c|}{.4} & \multicolumn{2}{|c|}{.5} & \multicolumn{2}{|c|}{.6} & \multicolumn{2}{|c|}{.7} & \multicolumn{2}{|c|}{ Overall } \\
\hline & Mean & $\mathrm{SD}$ & Mean & SD & Mean & $\mathrm{SD}$ & Mean & SD & Mean & SD & Mean & SD \\
\hline 160 & .04 & .04 & .13 & .10 & .55 & .13 & .86 & .06 & .95 & .06 & .51 & .38 \\
\hline 320 & .14 & .11 & .31 & .15 & .61 & .13 & .84 & .05 & .92 & .04 & .56 & .32 \\
\hline 480 & .14 & .07 & .32 & .08 & .63 & .09 & .81 & .10 & .89 & .09 & .56 & .30 \\
\hline 640 & .16 & .07 & .39 & .09 & .62 & .14 & .76 & .14 & .89 & .12 & .57 & .29 \\
\hline 800 & .15 & .08 & .31 & .11 & .61 & .10 & .80 & .12 & .90 & .10 & .55 & .31 \\
\hline 1000 & .12 & .07 & .33 & .08 & .60 & .14 & .80 & .11 & .85 & .13 & .54 & .30 \\
\hline 1200 & .09 & .04 & .30 & .12 & .53 & .13 & .70 & .50 & .78 & .12 & .48 & .28 \\
\hline 1500 & .19 & .08 & .39 & .14 & .56 & .20 & .70 & .16 & .79 & .17 & .53 & .26 \\
\hline Overall & .13 & .08 & .31 & .13 & .59 & .14 & .78 & .13 & .87 & .12 & .54 & .13 \\
\hline
\end{tabular}

The $T \times$ space and $T \times$ time interactions for the kappa experiment can be studied in Figure 5, which is a plot of mean proportions for each value of $T$. The figure shows the change in spatial influence on temporal judgments as $T$ was varied. For the two lowest $T$ values $(160$ and $320 \mathrm{msec})$, there was little separation between the $t_{1}$ curves, and the predominant kappa effects (positive slopes) seem to have resulted from the subjects' basing their temporal judgments almost entirely upon the spatial relationships. At intermediate values of $T(480,640$, and $800 \mathrm{msec})$, the subjects were able to make temporal judgments reasonably efficiently (reflected in the separation between the $t_{1}$ curves), although judgments continued to be influenced by the spatial characteristics of the display for all $t_{1} / T$ ratios. However, when $T$ was greater than or equal to $1,000 \mathrm{msec}$, the kappa effect was present only for the more difficult judgments (intermediate values of $t_{1} / T=.4, .5$, and .6). At the two extreme $t_{1} / T$ values $(.3$ and .7$)$, where there was little ambiguity about the temporal judgment, there was little or no spatial effect.

The results of the experiment demonstrate, as we have hypothesized, that the total display duration, $T$, is an important factor in the occurrence of the tau and kappa effects. The effect of $T$ is perhaps more marked for the kappa effect, although, as we have suggested, when $T$ is brief the effect of the temporal context on the spatial judgment is not apparent.

\section{EXPERIMENT 3}

On the basis of Experiment 2, we would argue that the tau and kappa effects are contextual illusions that are likely to arise when either primary judgment is made increasingly indeterminate. In this experiment, we shall try to provide a simple formal treatment of the context of the two illusions.

Several authors have suggested that a natural specification of the two illusions can be made in terms of imputed velocity (Anderson, 1974; Collyer, 1977; Price-Williams, 1954). Price-Williams argued that subjects may impute motion to the discontinuous displays, and Collyer (1977) noted that the tau and kappa effects would arise in relative judgment of two intervals if the subject were to set the ratio $s_{1} / t_{1}$ equal to the ratio $s_{2} / t_{2}$, which is formally equivalent to equalizing two velocities.

Anderson (1974) has suggested an algebraic model of the kappa effect based upon the assumption that subjects impute constant velocity (uniform) motion to the displays. The subject's response is essentially a weighted average of the time taken to traverse a given distance and of some expectation, $E(t)$, of the time that would be required to traverse the distance in uniform motion. The argument extends straightforwardly to the tau effect; judgments of distance are the weighted averages of the given distances and of the expected distances, $\mathrm{E}(\mathrm{s})$, which would be traversed in uniform motion in the given time. By hypothesis, $E(t)$ and $E(s)$ are linear functions of distance and duration, respectively, and we can write the subject's response, $R_{i j}$, to a pairing, $t_{i}$ and $s_{j}$, as the weighted mean of the scale values of the stimuli, that is,

$$
R_{i j}=w t_{i}^{*}+(1-w) s_{j}^{*}+e,
$$

where $w$ is a weight parameter such that $0<w<1$, e is an error term with zero expectation, and $t_{i}{ }^{*}$ and $s_{j}^{*}$ are scale values of the stimuli $t_{i}$ and $s_{j}$, respectively.

If the subject makes numerical (e.g., rating-scale) responses, Equation 1 implies that factorial pairings of $t_{i}$ and $s_{i}$ should result in mean judgments which plot as a set of linear parallel curves. The test of Equation 1 can therefore be made graphically or through the analysis of variance. The weight parameter, $w$, allows a simple treatment of the effect of the context on judgment. If, for example, the spatial context has no effect on temporal judgment, $1-w$ is set to zero. At the other extreme, temporal judgments may be so 


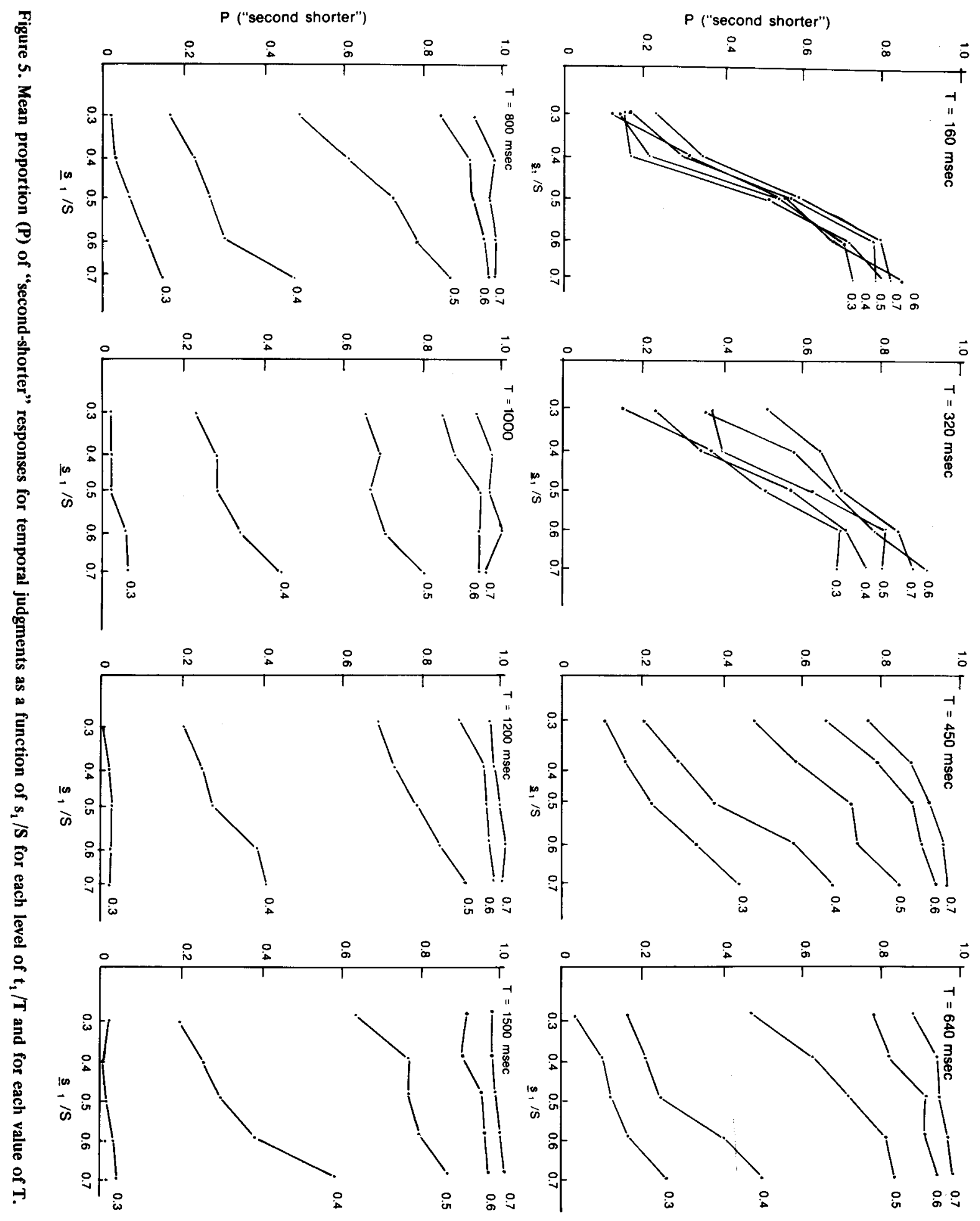


ambiguous that no weight is given to $t_{i}$ and temporal judgments are simply proportional to scale values of the $s_{i}$. Jones and Huang (1982) have shown that Equation 1 provides a satisfactory description of some kappa data (Price-Williams, 1954), and the purpose of the present experiment was to show that tau data can be described in the same way. The experiment also uses a sequence of eight lights so as to extend the demonstration of tau beyond the typical three-stimulus experiments.

\section{Method}

Subjects. The subjects were five undergraduates (three males and two females), who are paid $\$ 2 / \mathrm{h}$ for participation.

Apparatus. The apparatus was that used in Experiment 1.

Design and Procedure. The subjects were given practice in making absolute judgments of five distances, $2.67,3.33,4.00$, 4.67 , and $5.33 \mathrm{deg}$ of visual angle, which were assigned labels $1,2,3,4$, and 5 , respectively. During the initial 15 viewing trials, in which the five distances were presented three times at random, the phrase "LENGTH $=s$ " (where $x=1,2,3,4$, or 5 ) was first presented on the video screen for $1 \mathrm{sec}$. The corresponding distance was then indicated by two simultaneous lights for 500 msec. The subject initiated the next trial by pressing a response button.

Immediately following the viewing trials, the subject participated in an absolute judgment task. A trial began with the presentation of a distance, by means of the two lights, for $500 \mathrm{msec}$. The subject responded by pressing one of five buttons corresponding to the distance. No feedback was provided. The proportion of correct responses was computed following each block of 50 trials. The practice procedure was repeated until the subject attained between .50 and .65 accuracy.

In the experiment proper, a sequence of seven lights along the horizontal meridian was used. The lights defined six constant distances of .66 deg and six constant durations of $600 \mathrm{msec}$. An eighth light was used to define a variable time and distance at the end of the constant sequence. The five values of $s_{j}$ were those used in the practice sequence, and these values were factorially paired with five values of $t_{i}, 600,700,800,900$, and 1,000 msec.

Each trial proceeded with a "READY" message displayed on a video screen. The subject initiated a trial by pressing a response button, and $500 \mathrm{msec}$ later the display of eight lights was presented from left to right. The subject responded by pressing one of five response buttons corresponding to the distance. Each subject participated in two blocks of 1,000 trials on consecutive days.

\section{Results}

Figure 6 shows mean judgments of visual extent as a function of $\mathrm{t}_{\mathrm{i}}$. The plots are approximately linear, and the ANOVA showed significant effects of $s_{j}$ $[F(4,16)=72.40, p<.001]$ and $t_{i}[F(4,16)=6.52$, $p<.003]$ and the absence of a significant $t_{i} \times s_{j}$ interaction. The figure does show, however, despite the lack of significant interaction, that the topmost (5.33-deg) curve is relatively flat. Possibly, the subjects given the absolute judgment task used the largest spatial separation as a fixed standard, with the result that this value of $s_{j}$ was relatively little affected by the temporal factor.

In short, we have demonstrated a tau effect using absolute judgments and an eight-stimulus paradigm. Moreover, the results were in reasonable conformity to Equation 1.

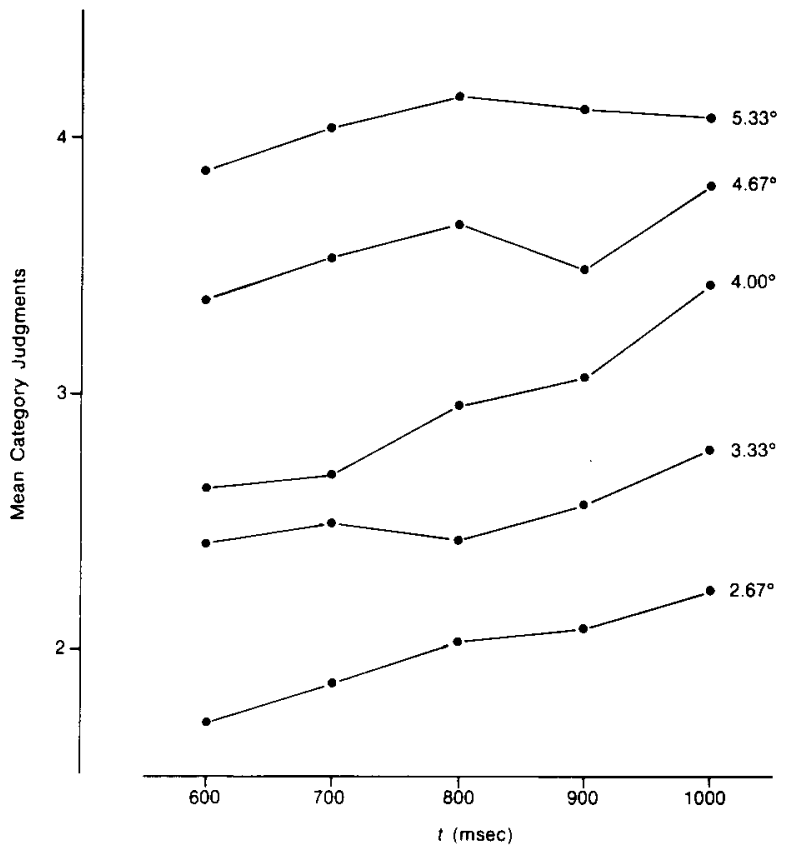

Figure 6. The tau effect. Mean judgments of length as a function of $t_{\mathbf{f}}$ for each value of $\mathbf{s}_{\mathbf{j}}$.

\section{CONCLUSIONS}

Experiment 1 confirms Collyer's (1977) finding that the tau and kappa effects are inconsistent either within or between subjects when $T$ is very brief, and Experiment 2 illustrates the general effect of variation in T, at least within the range of 160 to $1,500 \mathrm{msec}$, on both the tau and kappa effects. We have argued that $T$ is a crucial factor, since spatial and temporal discriminability may be expected to vary with $T$ and hence change the saliency of the context.

Our hypothesis that the two illusions should be more marked when the primary judgment is less determinate received detailed support for the kappa effect and significant, though less detailed, support for the tau effect. One important discrepancy remains. The tau effect in Experiment 2 was not apparent for easier spatial judgments when $s_{2}$ was the longer of the two extents but was apparent for easier judgments when $s_{2}$ was the shorter interval. Further work will be needed to explain this finding.

However, Experiment 3 does provide results for the tau effect which fit the assumption that the illusion may be described as a weighted average of the scale values of pairs of distances and durations. The context of the illusion can be described as an expectation of distance or duration derived from a formal imputation of uniform motion to the display. Equation 1 allows a convenient quantification of the saliency of the context (or, what amounts to the same 
thing, of the ambiguity of the primary judgment).

Given that Equation 1 passes the graphical or statistical test, Anderson (1974) has shown that the marginal means of the factorial design constitute interval scale representations of the stimuli. In other words, the distance between the curves in Figure 6 can be plotted against $s_{j}$ to form a psychophysical function. It is apparent that the curves are separated by roughly constant distances, implying a linear psychophysical function for spatial extent in conformity with the function established by other scaling methods (e.g., Teghtsoonian, 1965).

The majority of applications of Anderson's "functional measurement" (Anderson, 1974) have used rating-scale procedures for which an equation such as Equation 1 may be immediately valid. Many of the data on the tau and kappa effects, including the data of Experiments 1 and 2, are in the form of choice proportions which may require transformation to yield parallelism. The particular transformation would depend upon assumptions about the form of internal noise for the choice representation. Collyer (1977), for example, assumed Gaussian noise. We would, therefore, need to modify Equation 1, where appropriate, by writing,

$$
R_{i j}=F\left[w t_{i}^{*}+(1-w) s_{j}^{*}\right],
$$

where $F$ is, in Anderson's terms, a psychomotor function. The form of $F$ would determine the appropriate transformation to yield parallelism. A slightly different approach has been advocated by Collyer (1977), who suggests that relative judgments are statistical decisions based upon an internal code, $Y$, defined as the weighted sum of two random variables, one dependent upon the relative magnitudes of $s_{1}$ and $s_{2}$ and the other based upon the relative magnitudes of $t_{1}$ and $t_{2}$. The value of $Y$ is compared with a criterion value to produce a response on a given trial. This model is more general than Equation 1, since negative weights are allowed which would produce reverse tau or kappa effects.

Nevertheless, it remains difficult to specify the conditions under which negative weighting is likely, although we have had some success, particularly for the kappa effect, in specifying the conditions under which lower positive or zero weightings of the context should be apparent. Reverse tau or kappa effects do seem to be a feature of very brief perceptual displays, and one may argue, as does Collyer (1977), that a subject's strategies for combining spatial and temporal information tend to be idiosyncratic for brief T. Certainly, more work is needed to determine the range of individual differences in tau and kappa for $T$ less than about $200 \mathrm{msec}$ and to identify the determinants of such differences.

Overall, however, the present results are encouraging. A simple information integration model provides at least a first-order approximation to tau and kappa data (cf. Collyer, 1977), and there is striking evidence that the difficulty of the primary judgment predicts the degree to which subjects will rely upon the context.

\section{REFERENCES}

Allan, L. G. The perception of time. Perception \& Psychophysics, 1979, 26, 340-354.

Anderson, N. H. Algebraic models in perception. In E. C. Carterette \& M. P. Friedman (Eds.), Handbook of perception (Vol. 2). New York: Academic Press, 1974.

Bill, J. C., \& TEFT, L. W. Space-time relations: Effects of time on perceived visual extent. Journal of Experimental Psychology, 1969, 81, 196-199.

BILL, J. C., \& TEFT, L. W. Space-time relations: The effects of variations in stimulus and interstimulus interval duration on perceived visual extent. Acta Psychologica, 1972, 36, 358-369.

Cohen, J., Hansel, C. E. M., \& Sylvester, J. D. A new phenomenon in time judgment. Nature, 1953, 172, 901

Cohen, J., Hansel, C. E. M., \& Sylvesten, J. D. Interdependence of temporal and auditory judgments. Nature, 1954, $174,642$.

Cohen, J., Hansel, C. E. M., \& Sylvester, J. D. Interdependence in judgments of space, time and movement. Acta Psychologica, 1955, 11, 360-372.

Collyer, C. E. Discrimination of spatial and temporal intervals defined by three light flashes: Effects of spacing on temporal judgments and of timing on spatial judgments. Perception \& Psychophysics, 1977, 21, 357-364.

Crovitz, M. F., \& Daves, W. Tendencies to eye movements and perceptual accuracy. Journal of Experimental Psychology, 1962, 63, $495-498$.

Helson, H. The tau-effect. An example of psychological relativity. Science, 1930, 71, 536-537.

Helson, H., \& King, S. M. The tau-effect. An example of psychological relativity. Journal of Experimental Psychology, 1931, 14, 202-217.

Jones, B., \& Huang, Y. L. Space-time dependencies in psychophysical judgment: Algebraic models of the tau and kappa effects. Psychological Bulletin, 1982, 91, 128-142.

Kinchla, R. A., \& Smyzer, F. A diffusion model of perceptual memory. Perception \& Psychophysics, 1967, 2, 219-229.

Price-Williams, D. R. The kappa effect. Nature, 1954, 173, 363-364.

Teghtsoonian, M. The judgment of length. American Journal of Psychology, 1965, 73, 247-255.

(Manuscript received September 10, 1981; revision accepted for publication February 12, 1982.) 